\title{
Berberine attenuates myocardial ischemia reperfusion injury by suppressing the activation of PI3K/AKT signaling
}

\author{
ZHU QIN-WEI $^{1}$ and LI YONG-GUANG ${ }^{1,2}$ \\ ${ }^{1}$ Department of Emergency, Weifang Hospital of Traditional Chinese Medicine; ${ }^{2}$ Department of Emergency, \\ Weifang People's Hospital, Weifang, Shandong 261041, P.R. China
}

Received October 12, 2014; Accepted October 21, 2015

DOI: $10.3892 /$ etm.2016.3018

\begin{abstract}
Berberine (BBR), an isoquinoline alkaloid originally isolated from the Chinese herb Coptis chinensis (Huanglian), exhibits anti-inflammatory and immunosuppressive properties. Since myocardial ischemia/reperfusion (I/R) injury is associated with an excessive immune response, the current study was conducted to investigate the impact of BBR on myocardial I/R injury, a common disorder in clinical settings. Preconditioning of Sprague-Dawley rats with BBR (100 mg/kg/day, by gavage) for 14 days prior to the induction of I/R significantly attenuated myocardial I/R injury as manifested by a reduction in the incidence of ventricular arrhythmia and the amelioration of myocardial histological changes. These effects were found to be associated with the suppression of the phosphoinositide 3-kinase/AKT signaling pathway and the subsequent reduction of the expression of interleukin (IL)-6, IL-1 $\beta$, and tumor necrosis factor- $\alpha$ in the serum and myocardial tissue. These results indicate that BBR has the potential be an effective alternative therapy for the prevention and treatment of myocardial I/R injury in clinical practice.
\end{abstract}

\section{Introduction}

Myocardial ischemia/reperfusion (I/R) injury is a common pathological process in numerous clinical settings, including aortic bypass surgery, cardiopulmonary surgery and heart transplantation $(1,2)$. The exact molecular mechanisms underlying myocardial $\mathrm{I} / \mathrm{R}$ injury are complicated and are yet to be fully understood. However, there is considerable evidence that inflammation has an important role in myocardial I/R injury $(3,4)$. Notably, the phosphoinositide 3-kinase (PI3K)/AKT signaling pathway, which was originally recognized to have a critical role in the regulation of cell growth and

Correspondence to: Dr Yong-Guang Li, Department of Emergency, Weifang People's Hospital, 151 Guang Wen Road, Weifang, Shandong 261041, P.R. China

E-mail: 1476284406@qq.com

Key words: myocardial ischemia reperfusion injury, inflammation, berberine, phosphoinositide 3-kinase, protein kinase B cell survival in various systems $(5,6)$, has been shown to be involved in the protection of the kidneys and liver against I/R injury by regulating the inflammatory response $(7,8)$.

The PI3K enzyme consists of a catalytic subunit p110 and a regulatory subunit $\mathrm{p} 85$, and its activation relies on $\mathrm{p} 85$ phosphorylation. Once p85 has been activated, it initiates the phosphorylation of Akt (also known as protein kinase B), which subsequently triggers signaling pathways in order to increase the inflammatory response $(9,10)$. Inhibiting the activation of the PI3K/AKT signaling pathway has been demonstrated to attenuate I/R-induced injury (7).

Berberine (BBR) is an isoquinoline alkaloid compound that was originally isolated from the Chinese herb Coptis chinensis (Huanglian) (11). It is an antimicrobial drug routinely prescribed for the treatment of diarrhea in various Asian countries (11). In addition to this well-known and widely recognized effect, it has been demonstrated that BBR also regulates the activity of transcription factors essential for the inflammatory response (12). BBR has been shown to provide intestinal and cerebral protection against I/R-induced injury $(13,14)$; however, the effects of BBR against myocardial I/R injury are yet to be elucidated. Therefore, the present study investigated whether BBR was able to prevent myocardial I/R injury in a rat model.

\section{Materials and methods}

Animals. A total of 50 male Sprague Dawley (SD) rats $(200-250 \mathrm{~g})$ were purchased from the Hua Fukang Experimental Animal Center (Beijing, China). The rats were housed in a specific pathogen-free facility at $18-29^{\circ} \mathrm{C}$ under a $14 \mathrm{~h} \mathrm{light} / 10 \mathrm{~h}$ dark cycle, and were fed with laboratory chow and water. After a minimum 7 days of acclimation, the rats were randomly divided into five groups as follows (10 rats/group): i) The I/R injury (IRI) group, in which saline-treated rats were subjected to ischemia for $0.5 \mathrm{~h}$ followed by reperfusion; ii) three BBR groups in which the rats were treated with BBR at doses of 25,50 or $100 \mathrm{mg} / \mathrm{kg} /$ day, respectively, by gavage 14 days prior to the induction of I/R; and iii) a sham group, in which saline-treated rats were subjected to sham surgery without the induction of ischemia. The dosages administered to the rats were determined according to a previous study (15). All experiments were approved by the Institutional Animal Care and Use Committee at Weifang People's Hospital (Weifang, China). 
Induction of myocardial I/R. The rats were anesthetized with intraperitoneal injection of $1 \%$ sodium pentobarbital solution $(65 \mathrm{mg} / \mathrm{kg})$ and their temperature was regulated throughout the test by means of a heating lamp. Myocardial I/R was induced as follows: The rats were placed in the supine position and secured in a dissection tray. A left thoracic incision was made to expose the heart, and myocardial ischemia was induced by making a slipknot (4-0 silk) around the left anterior descending coronary artery. After $30 \mathrm{~min}$ of ischemia, the slipknot was released and the myocardium was reperfused for $4 \mathrm{~h}$. The rats in the sham group underwent the same surgical procedures with the exception of occlusion of the left anterior descending coronary artery. Myocardial function was continuously monitored prior to and during the I/R procedure and during the ischemia and reperfusion period with an electrocardiogram (ECG) recorder (ECG-9020p; Nihon Kohden, Tokyo, Japan). The total time of recording was $4.5 \mathrm{~h}$.

The incidence and count of premature ventricular contraction (PVC) and the incidence and cumulative duration of ventricular tachycardia (VT) and ventricular fibrillation (VF) were recorded. Ventricular arrhythmia (VA) was scored using the criteria described by Curtis and Walker (16) and Ravingerova et al (17).

Histological analysis. At $3 \mathrm{~h}$ of reperfusion, the rats were anesthetized with intraperitoneal injection of $1 \%$ sodium pentobarbital solution $(65 \mathrm{mg} / \mathrm{kg})$, after which they were sacrificed by cervical dislocation in order to collect serum and myocardial tissue. The excised hearts were fixed with $10 \%$ formalin, embedded in paraffin, and stained with hematoxylin and eosin (H\&E). Myocardial I/R injury was scored using published morphologic criteria (18): 0 , no damage; 1 (mild), interstitial edema and localized necrosis; 2 (moderate), widespread myocardial cell swelling and necrosis; 3 (severe), necrosis with contraction bands and compressed capillaries, or 4 (highly severe), diffuse necrosis with contraction bands, compressed capillaries and hemorrhage.

Western blot analysis. Myocardial samples (50 mg) were mechanically homogenized in $1 \mathrm{ml}$ hypotonic buffer, containing $200 \mu$ 1 4-(2-hydroxyethyl)-1-piperazineethanesulfonic acid ( $\mathrm{pH} 7.5$ ), $25 \mathrm{mmol} / \mathrm{l}$ magnesium chloride, $5 \mathrm{mmol} / \mathrm{l}$ dithiothreitol, $5 \mathrm{mmol} / \mathrm{l}$ phenylmethylsulfonyl fluoride, $2 \mathrm{mmol} / \mathrm{l}$ pepstatin A, $10 \mu \mathrm{g} / \mathrm{ml}$ leupeptin, $5 \mathrm{mmol} / \mathrm{l}$ ethylenediamine tetraacetic acid, $10 \mu \mathrm{g} / \mathrm{ml}$ aprotinin and $100 \mu \mathrm{l}$ anti-phosphatases (Roche Diagnostics, Basel, Switzerland). Protein concentrations were determined using a bicinchoninic assay kit (Biyuntian Biotechnology, Wuhan, China), according to the manufacturer's protocol. Protein samples $(80 \mu \mathrm{g})$ were resolved by $12 \%$ sodium dodecyl sulfate-polyacrylamide gel electrophoresis and transferred to polyvinylidene fluoride membranes (San Yin-tan, Beijing, China). The membranes were blocked with $5 \%$ non-fat skimmed milk in Tris-buffered saline solution containing Tween-20 (TBST; $10 \mathrm{mmol} / \mathrm{l}$ Tris, $\mathrm{pH} 7.5 ; 140 \mathrm{mmol} / 1$ sodium chloride; $0.1 \%$ Tween-20) for $1 \mathrm{~h}$ at $37^{\circ} \mathrm{C}$, washed and then incubated with primary antibody in TBST containing 3\% bovine serum albumin (Gibco; Thermo Fisher Scientific, Inc., Waltham, MA, USA) for $12 \mathrm{~h}$ at $4^{\circ} \mathrm{C}$. The primary antibodies were as follows: Rabbit anti-p85 (1:1,000; 4292), rabbit anti-phosphorylated (p)-p85 (1:500;
4228), rabbit anti-Akt $(1: 1,000 ; 4691)$ and rabbit anti-p-Akt (1:500; 4060) (Cell Signaling Technology, Inc., Danvers, MA, USA). The membranes were then washed extensively with TBS, prior to incubation with a horseradish peroxidase-conjugated immunoglobulin G secondary antibody (1:4,000; J115005072; Jackson ImmunoResearch Laboratories, West Grove, PA, USA) for $1 \mathrm{~h}$ at $37^{\circ} \mathrm{C}$. $\beta$-actin $(1: 3,000 ; 113257$; Abmart, Shanghai, China) was used for the normalization of protein levels. The reactive bands were visualized using the Enhanced Chemiluminescence-Plus reagent (GE Healthcare Life Sciences, Piscataway, NJ, USA) according to the protocol provided by the manufacturer. The density of each reactive band was quantified using the LabWorks Image Acquisition platform (UVP, Inc., Upland, CA, USA), and ImageJ (National Institutes of Health, Bethesda, MA, USA).

Enzyme-linked immunosorbent assay (ELISA) analysis. At $3 \mathrm{~h}$ of reperfusion, the rats were anesthetized with intraperitoneal injection of $1 \%$ sodium pentobarbital solution $(65 \mathrm{mg} / \mathrm{kg})$, after which they were sacrificed for collection of serum and myocardial tissue. Levels of the inflammatory mediators tumor necrosis factor (TNF)- $\alpha$, interleukin (IL)- 6 and IL-1 $\beta$ in the serum were quantified using specific ELISA kits for rats, according to the manufacturer's protocol (BioSource ${ }^{\mathrm{TM}}$; Thermo Fisher Scientific, Waltham, MA, USA).

Reverse transcription-quantitative polymerase chain reaction $(R T-q P C R)$ analysis. Total RNA was isolated from myocardial tissues using TRIzol ${ }^{\circledR}$ reagent, according to the manufacturer's protocol (Thermo Fisher Scientific, Inc.). Total RNA (4 $\mu \mathrm{g})$ was reverse transcribed into cDNA using the PrimeScript SYBR Premix Ex Taq ${ }^{\mathrm{TM}}$ II (PerfectReal Time; Takara Bio, Inc., Otsu, Japan), as instructed by the manufacturer. qPCR amplifications were conducted using the ABI 7500 Real Time PCR system (Applied Biosystems; Thermo Fisher Scientific). PCR primers (Invitrogen; Thermo Fisher Scientific) for all analyzed mRNA are presented in Table I. PCR was conducted at $95^{\circ} \mathrm{C}$ for $30 \mathrm{sec}$, followed by 40 cycles at $95^{\circ} \mathrm{C}$ for $5 \mathrm{sec}, 60^{\circ} \mathrm{C}$ for $34 \mathrm{sec}$ and $95^{\circ} \mathrm{C}$ for $15 \mathrm{sec}$. The amount of mRNA for each gene was normalized using $\beta$-actin, and the relative expression levels were calculated using the $2^{-\Delta \Delta \mathrm{Cq}}$ method, as previously reported (19).

Statistical analysis. Data are presented as the mean \pm standard error of the mean. Comparisons between groups were performed using the Student's t-test or one-way analysis of variance. $\mathrm{P}<0.05$ was considered to indicate a statistically significant difference.

\section{Results}

$B B R$ attenuates the $I / R$-induced incidence of ventricular arrhythmia. The impact of BBR on ventricular arrhythmia following I/R insult was first investigated. For this purpose, the incidence and count of PVC and the incidence and cumulative duration of VT and VF were examined, and VA scores were determined. As compared with the rats in the sham group, rats in the IRI group exhibited an increased incidence and count of PVC, increased incidence and cumulative duration of VT and VF and higher arrhythmia scores $(\mathrm{P}<0.05)$, indicating that $\mathrm{I} / \mathrm{R}$ insult induced severe ventricular arrhythmia. Notably, rats treated with BBR had a lower incidence and count/cumula- 
Table I. Primers used for quantitative polymerase chain reaction analysis.

\begin{tabular}{lcll}
\hline mRNA & Species & \multicolumn{1}{c}{ Forward } & \multicolumn{1}{c}{ Reverse } \\
\hline TNF- $\alpha$ & Rat & CTGAACTTCGGGGTGATCGG & GGCTTGTCACTCGAATTTTGAGA \\
IL-6 & Rat & AGCTTCCTTGTGCAAGTGTCT & GACAGCCCAGGTCAAAGGTT \\
IL-1 $\beta$ & Rat & CTGCAAGAGACTTCCATCCAG & AGTGGTATAGACAGGTCTGTTGG \\
$\beta$-actin & Rat & AGAGGGAAATCGTGCGTGAC & CAATAGTGATGACCTGGCCGT \\
\hline
\end{tabular}

TNF, tumor necrosis factor; IL, interleukin.

Table II. Effects of BBR on arrhythmia in IRI model rats.

\begin{tabular}{|c|c|c|c|c|c|c|c|c|}
\hline \multirow[b]{2}{*}{ Group } & \multirow[b]{2}{*}{ Number } & \multicolumn{2}{|c|}{$\mathrm{PVC}$} & \multicolumn{2}{|c|}{ VT } & \multicolumn{2}{|c|}{ VF } & \multirow[b]{2}{*}{ VA score } \\
\hline & & Incidence & Count & Incidence & Duration (sec) & Incidence & Duration (sec) & \\
\hline Sham & 10 & 50 & $9 \pm 11$ & 0 & 0 & 0 & 0 & $0.4 \pm 0.5$ \\
\hline IRI & 10 & 80 & $37 \pm 22^{\mathrm{a}}$ & 60 & $27.2 \pm 24.6^{\mathrm{a}}$ & 40 & $15.5 \pm 17.2^{\mathrm{a}}$ & $5.0 \pm 4.3^{\mathrm{a}}$ \\
\hline BBR $(25 \mathrm{mg} / \mathrm{kg})$ & 10 & 80 & $32 \pm 21^{\mathrm{b}}$ & 50 & $22.7 \pm 26.6^{\mathrm{b}}$ & 40 & $11.5 \pm 14.7^{\mathrm{b}}$ & $4.2 \pm 4.1^{\mathrm{b}}$ \\
\hline BBR (50 mg/kg) & 10 & 60 & $21 \pm 15^{\mathrm{b}}$ & 30 & $10.8 \pm 16.6^{\mathrm{b}}$ & 15 & $5.6 \pm 7.8^{\mathrm{b}}$ & $2.5 \pm 3.2^{\mathrm{b}}$ \\
\hline BBR $(100 \mathrm{mg} / \mathrm{kg})$ & 10 & 60 & $15 \pm 12^{\mathrm{b}}$ & 20 & $3.4 \pm 7.0^{\mathrm{b}}$ & 5 & $1.8 \pm 4.2^{\mathrm{b}}$ & $1.5 \pm 3.0^{\mathrm{b}}$ \\
\hline
\end{tabular}

Results were obtained by the analysis of electrocardiograms. BBR, berberine; IRI, ischemia/reperfusion injury; PVC, pre-ventricular contraction; VF, ventricular fibrillation; VT, ventricular tachycardia; VA, ventricular arrhythmia. Values are presented as the mean \pm standard deviation. ${ }^{a} \mathrm{P}<0.05$ vs. the sham group. ${ }^{\mathrm{b}} \mathrm{P}<0.05$ vs. the IRI group.

tive duration of PVC, VT and VF, and reduced arrhythmia scores compared with those of the rats in the IRI group, and the values were reduced in a dose-dependent manner $(\mathrm{P}<0.05$; Table II). These data indicate that the administration of BBR protected the rats against I/R-induced myocardial injury and the greatest protective effect was observed at a BBR concentration of $100 \mathrm{mg} / \mathrm{kg}$.

$B B R$ attenuates $I / R$-induced pathological changes in the myocardial tissue. When the myocardial tissues from the IRI group were compared with those from the sham group, significant pathological changes were evident in the myocardial tissues from the IRI group (Fig. 1A and B). The changes observed in the IRI group included atrophy of myocardial fibers, inflammatory cell infiltration, coagulative necrosis and liquefactive necrosis. By contrast, the myocardial tissue in the BBR group (100 mg/kg) exhibited fewer pathological changes, as compared with the IRI group (Fig. 2C). Semi-quantitative assessment of the histological lesions showed a significantly higher score in the rats of the IRI group than in the rats of the sham and BBR groups (Fig. 2D).

BBR suppresses the activation of PI3K/AKT signaling. After ascertaining the concentration of BBR with the strongest protective effect against myocardial I/R injury, the next step was to explore the mechanisms underlying the protective effect of BBR against I/R-induced myocardial injury. It has previously been demonstrated that PI3K/AKT plays an important role in myocardial injury (15) and that BBR is able to regulate PI3K/AKT signaling (15). To this end, the activity of the PI3K p85 regulatory subunit was first investigated. No significant difference was detected in the expression of total p85 among the three groups of rats (Fig. 2). However, much higher levels of activated p85 (p-p85) were noted in the rats of the IRI group as compared with those in rats of either the BBR group or the sham group (Fig. 2). Notably, rats in the BBR group showed very similar levels of p-p85 to those in the sham group. Since p85 activation provides signals for AKT phosphorylation, AKT activity was then examined. Similar to $\mathrm{p} 85$, there was no difference in the levels of total AKT among the groups (Fig. 2), but activated AKT (p-AKT) levels were significantly higher in the rats of the IRI group, and rats in the BBR group displayed p-AKT levels very similar to those of the sham group (Fig. 2). Collectively, these data suggest that BBR pretreatment can decrease PI3K p85 activity, which results in the upregulation of AKT activation.

Blocking the activation of PI3K/AKT signaling suppresses inflammatory cytokine expression. In order to investigate whether blocking the activation of PI3K/AKT signaling suppressed the expression of inflammatory cytokines in the heart, the expression of TNF- $\alpha$, IL- 6 and IL- $1 \beta$ in cardiac tissues after I/R insult was analyzed by RT-qPCR. It was noted that $\mathrm{I} / \mathrm{R}$ insult increased the expression level of TNF- $\alpha$ by 9 -fold (Fig. 3A), IL-1 $\beta$ by 3.75 -fold (Fig. 3B) and IL-6 by 5 -fold (Fig. 3C) as compared with that of rats in the sham group. Notably, the administration of BBR inhibited I/R-induced TNF- $\alpha$ expression by $49 \%$ (Fig. 3A), IL-1 $\beta$ by $40 \%$ (Fig. 3B) and IL-6 by $42 \%$ (Fig. 3C). These data support the hypothesis that the attenuation of PI3K/AKT signaling 


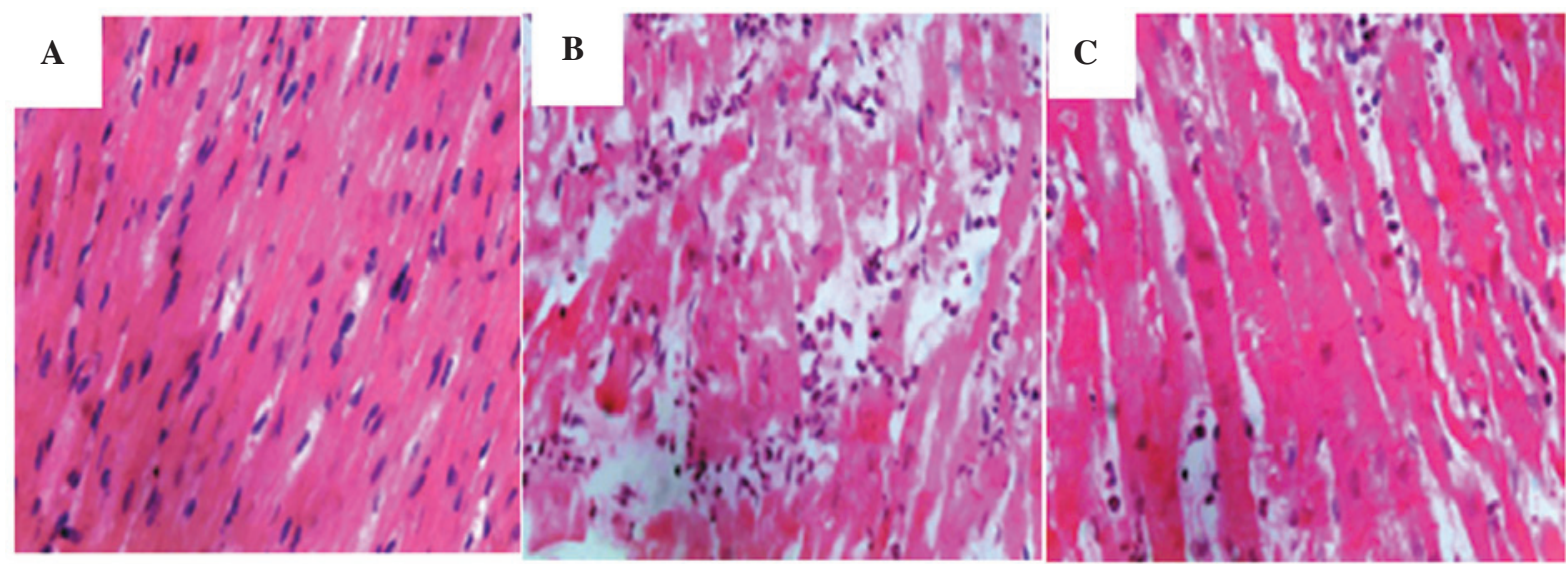

D

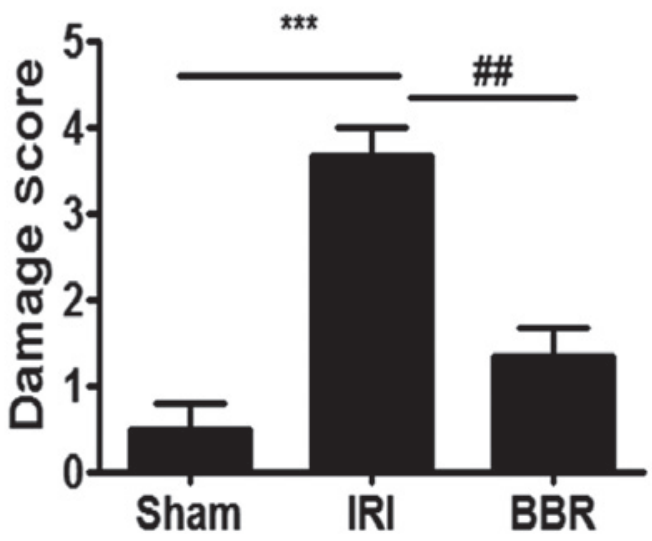

Figure 1. Histological analysis of myocardial tissues. Representative hematoxylin and eosin (H\&E) staining results for myocardial sections in rats of the (A) sham (B) ischemia-reperfusion injury (IRI) and (C) berberine (BBR) groups (magnification, $\mathrm{x} 200$ ). (D) Semi-quantitative analysis of $\mathrm{H} \& \mathrm{E}$ staining in the rats $\left(\mathrm{n}=3\right.$ per group). ${ }^{* * *} \mathrm{P}<0.001,{ }^{\# \#} \mathrm{P}<0.01$.
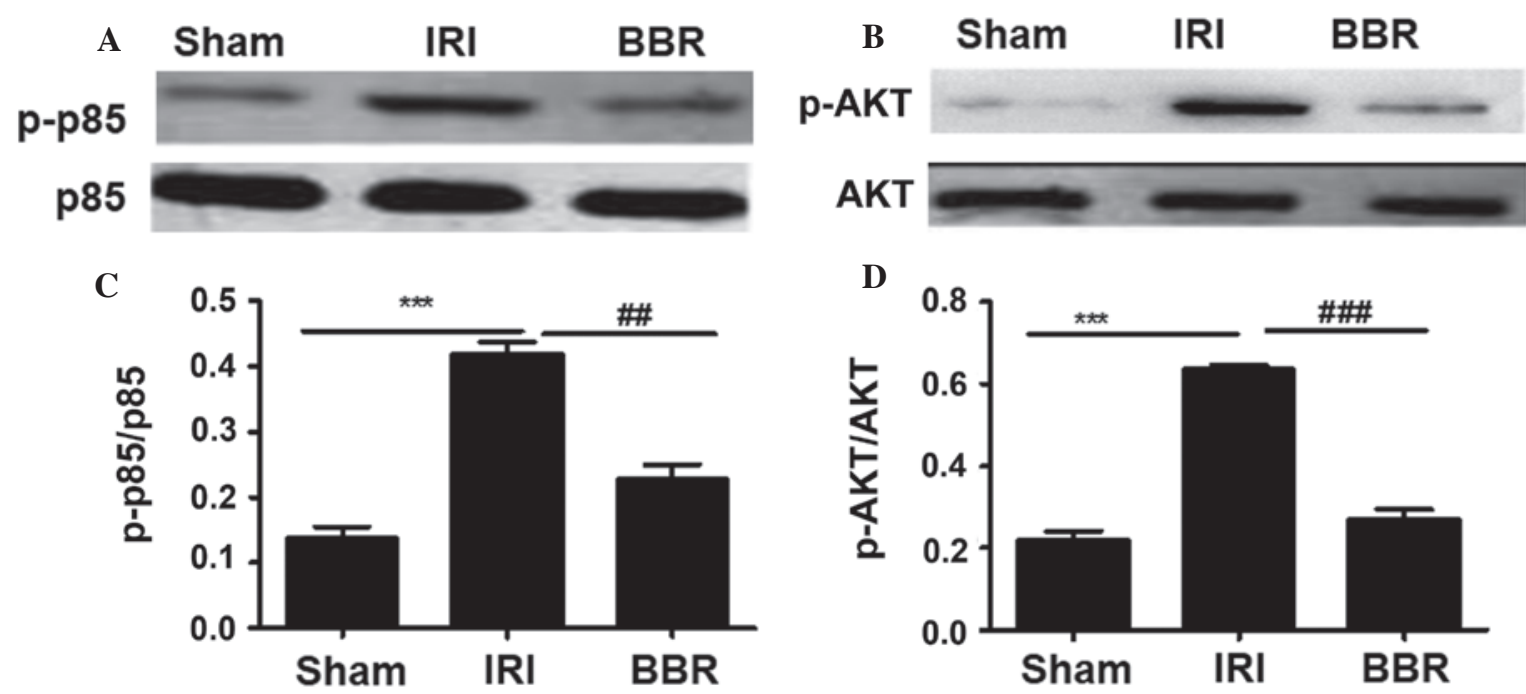

Figure 2. Administration of BBR inhibited the activation of PI3K/AKT signaling. The phosphorylated proteins were measured by western blotting to assess the activity of p85 and AKT. Representative western blots for (A) p85 and p-p85 and (B) AKT and p-AKT. Quantitative analysis of (C) p-p85 and (D) AKT activities. The relative activity for $\mathrm{p} 85$ and AKT was assessed as a ratio between the phosphorylated form and all forms of the protein (n=10 rats/group). ${ }^{* * *} \mathrm{P}<0.001$, ${ }^{\# \#} \mathrm{P}<0.01 ;{ }^{\# \#} \mathrm{P}<0.001$. BBR, berberine; IRI, ischemia/reperfusion injury; PI3K, phosphoinositide 3-kinase; p, phospho.

by the administration of BBR significantly suppressed I/R-induced inflammatory cytokine expression in the myocardial tissues.
Blocking the activation of PI3K/AKT signaling suppresses inflammatory cytokine secretion. In order to further elucidate the effect of BBR on the expression of inflammatory cytokines 

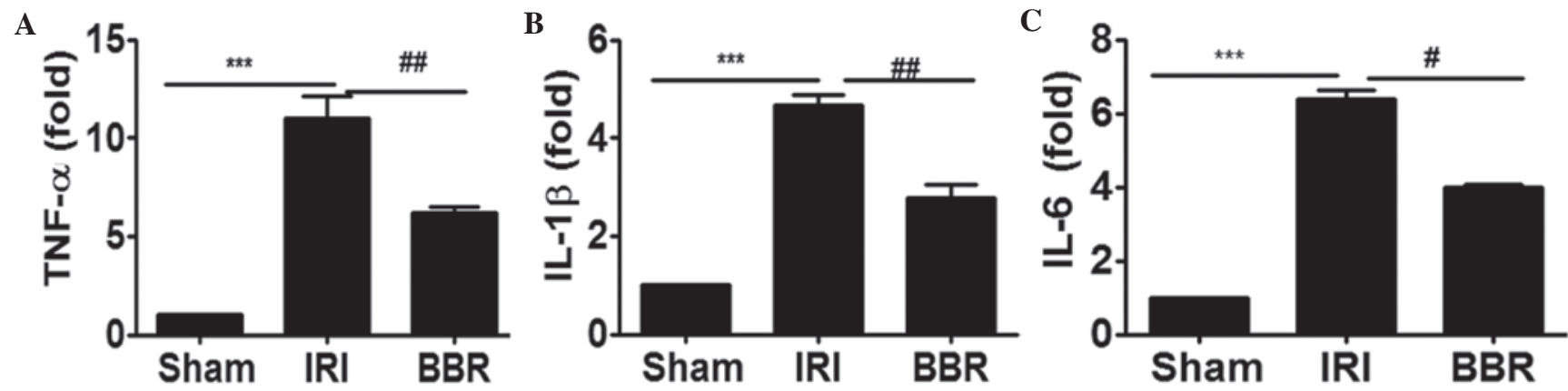

Figure 3. BBR treatment suppresses I/R-induced cytokine expression in the myocardium. Reverse transcription-quantitative polymerase chain reaction was employed to evaluate the expression of inflammatory cytokines in the myocardial tissue. The expression levels of (A) TNF- $\alpha$, (B) IL-1 $\beta$ and (C) IL-6 were examined after $4 \mathrm{~h}$ of myocardial I/R insult. ${ }^{* * *} \mathrm{P}<0.001,{ }^{\# \#} \mathrm{P}<0.01,{ }^{\#} \mathrm{P}<0.05$. Ten rats were included in each study group. BBR, berberine; I/R, ischemia/reperfusion; IRI, I/R injury; TNF, tumor necrosis factor; IL, interleukin.

A

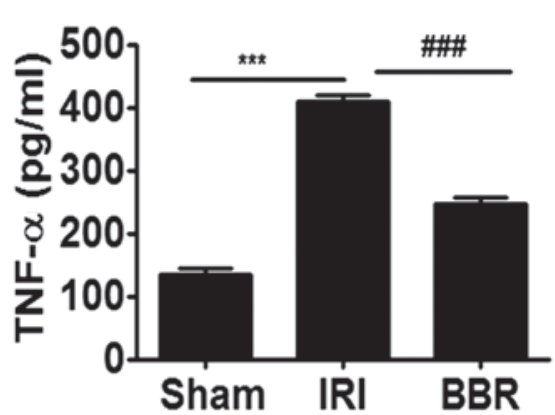

B

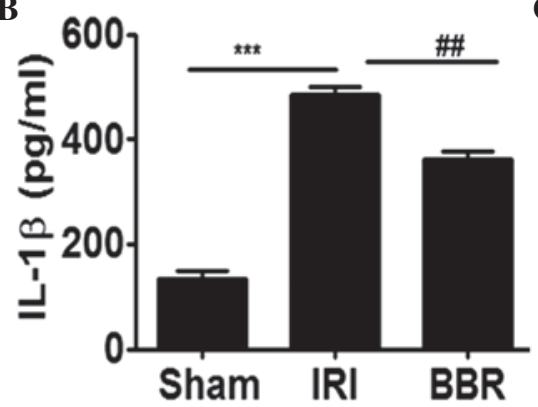

C

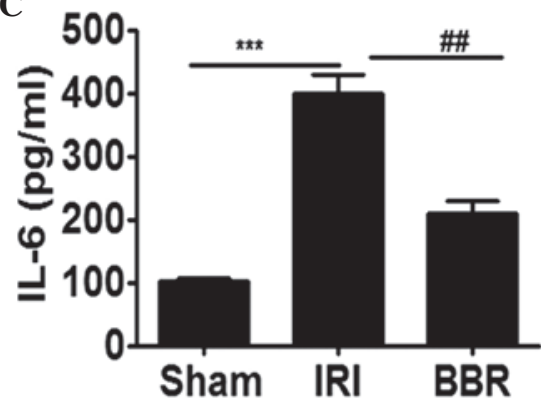

Figure 4. BBR treatment suppresses IR-induced cytokine expression in the serum. ELISA was employed to assess the expression of inflammatory cytokines in the serum. The expression levels of (A) TNF- $\alpha$, (B) IL-1 $\beta$ and (C) IL-6 were determined after $4 \mathrm{~h}$ myocardial I/R insult. ${ }^{* * *} \mathrm{P}<0.001,{ }^{\# \#} \mathrm{P}<0.01,{ }^{\# \#} \mathrm{P}<0.001$. Ten rats were included in each study group. BBR, berberine; I/R, ischemia/reperfusion; IRI, I/R injury; TNF, tumor necrosis factor; Il, interleukin; ELISA, enzyme-linked immunosorbent assay.

following I/R insult, the expression levels of TNF- $\alpha$, IL-6 and IL-1 $\beta$ in the serum were analyzed. It was noted that $\mathrm{I} / \mathrm{R}$ insult increased the secretion of TNF- $\alpha$ by 2 -fold (Fig. 4A), IL-1 $\beta$ by 2.5-fold (Fig. 4B) and IL-6 by 3-fold (Fig. 4C) as compared with that in the sham group. Notably, administration of BBR inhibited I/R-induced TNF- $\alpha$ expression by $45 \%$ (Fig. 4A), IL- $1 \beta$ by $35 \%$ (Fig. 4B) and IL-6 by $45 \%$ (Fig. 4C), and. These data indicate that the downregulation of PI3K/AKT signaling by BBR significantly suppressed I/R-induced inflammatory cytokine secretion in the serum.

\section{Discussion}

Although BBR has long been used for the treatment of diarrhea in a number of Asian countries (11), its exact impact on myocardial I/R injury, remains unclear and was thus investigated in the present study. I/R injury was induced in rats by blocking the left descending coronary artery for $30 \mathrm{~min}$, followed by reperfusion for $4 \mathrm{~h}$. ECG analysis demonstrated that myocardial I/R injury significantly increased the incidence and count of PVC, the incidence and cumulative duration of VT and VF, and arrhythmia scores. However, pretreatment with $\operatorname{BBR}(25,50$ or $100 \mathrm{mg} / \mathrm{kg} /$ day) by gavage for 14 days prior to the induction of I/R significantly attenuated the changes in ECG results in a dose-dependent manner. Moreover, pretreatment with BBR also decreased the IRI-induced histological changes of the myocardium as manifested by reductions in the atrophy of myocardial fibers, inflammatory cell infiltration, coagulative necrosis and liquefactive necrosis in the BBR group compared with those in the IRI group. These results are consistent with those of previous studies, which suggest that the treatment of rats with BBR can significantly decrease myocardial I/R injury and the subsequent induction of ventricular arrhythmias and myocardial histological changes $(15,20,21)$. The present data support the potential of BBR as a new therapeutic agent for the prevention and treatment of myocardial I/R injury in clinical practice.

To ascertain the molecular mechanisms by which BBR provides protection against myocardial $I / R$ injury in rats, the impact of BBR on PI3K/AKT signaling was investigated. I/R insult induced activation of the PI3K p85 regulatory subunit as manifested by the significantly higher levels of p-p85 in rats of the IRI group as compared with those in the sham control rats. BBR pretreatment significantly decreased the activation of $\mathrm{p} 85$. This result prompted the examination of AKT activity since the phosphorylation of p85 would predispose AKT to activation. Consistent with the aforementioned results, BBR significantly attenuated AKT activity as manifested by a reduction in the levels of p-AKT by more than half. To further investigate whether the suppression of PI3K/AKT signaling activation by BBR inhibited the inflammatory response, TNF- $\alpha$, IL-6 and IL-1 $\beta$ expression in the I/R-insulted myocardial tissues and serum were examined. Rats preconditioned with BBR displayed significantly lower levels of TNF- $\alpha$, IL-6 and IL- $1 \beta$ in myocardial tissue and serum than were observed in the saline-treated 
IRI group. These data suggest that BBR suppressed the activation of PI3K/AKT signaling, which then repressed the inflammatory response to mitigate myocardial I/R injury.

The duration of ischemia and time at which measurements are taken after reperfusion are critical factors relevant to the severity of $\mathrm{I} / \mathrm{R}$ injury in rats (1). Published data describe variations in results when experimental conditions such as temperature and duration of ischemia are changed.In particular, the results may vary depending on the strain of rats employed, for example SD versus diabetic rats (21). In the experimental model used in the present study, SD rats were employed, and a time period of $30 \mathrm{~min}$ was used for ischemic insult and I/R injury was examined $4 \mathrm{~h}$ after reperfusion. The degree of severity of the myocardial injuries in this model was noted to be similar to that in a previous study (15). As aforementioned, evidence for myocardial injury was strongly supported by the chamges in the electrocardiograms along with inflammatory infiltration. These data support the hypothesis that I/R initiates a complex cascade of events, which eventually result in myocardial injury characterized by changes in ECG results and inflammatory infiltration.

The PI3K/AKT pathway is known to be important in regulating the adaptive immune response. For example, PI3K heterodimers control cell survival, proliferation, B- and T-cell receptor signaling, and chemotaxis in B and $\mathrm{T}$ lymphocytes $(21,22)$. The PI3K/AKT signaling pathway also has a variety of roles in innate immune cells, including neutrophils, mast cells, monocytes, macrophages and myeloid as well as plasmacytoid dendritic cells. For example, the migration of innate immune cells into sites of injury in tissues or organs involves the dynamic reorganization of cytoskeletons and membrane structures, while PI3K signaling is essential for this process by providing cell polarity and pseudopodia extension (22). The hypothesis that the inhibition of PI3K attenuates I/R-induced myocardial injury has been investigated previously (23). Therefore, in the current study, no further experiments to demonstrate that the suppression of PI3K/AKT signaling attenuated the I/R-induced immune response in the myocardial tissue were conducted. Given the capacity of BBR preconditioning to prevent myocardial I/R injury, it is worthy of note that BBR may have an effect on other pathways in addition to the PI3K/AKT signaling pathway, for example, the MAPK kinase cascade (24). Further studies are required to investigate the pathways associated with $\mathrm{I} / \mathrm{R}$ insult.

In conclusion, the present study provides evidence that the preconditioning of rats with BBR protects against I/R-induced myocardial injury in a dose-dependent manner, as manifested by reduced ventricular arrhythmia and suppressed inflammatory infiltration. The mechanistic investigation demonstrated that BBR inhibits the activation of PI3K/AKT signaling, which then suppresses inflammatory infiltration and protects against myocardial I/R injury. These results support the use of BBR as an effective alternative therapy for the prevention and treatment of myocardial I/R injury in clinical practice.

\section{Acknowledgements}

The present study was supported by the National Natural Science Foundation of China (grant no. 81100164).

\section{References}

1. Chen K, Li G, Geng F, Zhang Z, Li J, Yang M, Dong L and Gao F: Berberine reduces ischemia/reperfusion-induced myocardial apoptosis via activating AMPK and PI3K-Akt signaling in diabetic rats. Apoptosis 19: 946-957, 2014.

2. Yan X, Qiu W, Jia B, Zhong H, Li X and Chen Z: Myocardial protection by interferon- $\gamma$ late preconditioning during cardiopulmonary bypass-associated myocardial ischemia-reperfusion in pigs. Oncol Rep 30: 2145-2152, 2013.

3. Lassaletta AD, Elmadhun NY, Zanetti AV, Feng J, Anduaga J, Gohh RY, Sellke FW and Bianchi C: Rapamycin treatment of healthy pigs subjected to acute myocardial ischemia-reperfusion injury attenuates cardiac functions and increases myocardial necrosis. Ann Thorac Surg 97: 901-907, 2014.

4. Du X, Hu X and Wei J: Anti-inflammatory effect of exendin-4 postconditioning during myocardial ischemia and reperfusion. Mol Biol Rep 41: 3853-3857, 2014.

5. Wu B, Meng K, Ji Q, Cheng M, Yu K, Zhao X, Tony H, Liu Y, Zhou Y, Chang C, et al: Interleukin-37 ameliorates myocardial ischaemia/reperfusion injury in mice. Clin Exp Immunol 176: 438-451, 2014.

6. Pourrajab F, Babaei Zarch M, Baghi Yazdi M, Rahimi Zarchi A and Vakili Zarch A: Application of stem cell/growth factor system, as a multimodal therapy approach in regenerative medicine to improve cell therapy yields. Int J Cardiol 173: 12-19, 2014.

7. Westin JR: Status of PI3K/Akt/mTOR pathway inhibitors in lymphoma. Clin Lymphoma Myeloma Leuk 14: 335-342, 2014.

8. Zhang J, Yao Y, Xiao F, Lan X, Yu C, Zhang Y, Jiang C, Yang J, Pei G, Li Y, et al: Administration of dexamethasone protects mice against ischemia/reperfusion induced renal injury by suppressing PI3K/AKT signaling. Int J Clin Exp Pathol 6: 2366-2375, 2013

9. Kim HJ, Joe Y, Kong JS, Jeong SO, Cho GJ, Ryter SW and Chung HT: Carbon monoxide protects against hepatic ischemia/reperfusion injury via ROS-dependent Akt signaling and inhibition of glycogen synthase kinase $3 \beta$. Oxid Med Cell Longev 2013: 306-421, 2013.

10. Hofmann BT and Jucker M: Activation of PI3K/Akt signaling by n-terminal SH2 domain mutants of the p $85 \alpha$ regulatory subunit of PI3K is enhanced by deletion of its c-terminal SH2 domain. Cell Signal 24: 1950-1954, 2012.

11. Tie G, Yan J, Yang Y, Park BD, Messina JA, Raffai RL, Nowicki PT and Messina LM: Oxidized low-density lipoprotein induces apoptosis in endothelial progenitor cells by inactivating the phosphoinositide 3-kinase/Akt pathway. J Vasc Res 47: 519-530, 2010.

12. Wang F, Yang Y, Ma LL, Tian XJ and He YQ: Berberine ameliorates renal interstitial fibrosis induced by unilateral ureteral obstruction in rats. Nephrology (Carlton) 19: 542-551, 2014.

13. Cui G, Qin X, Zhang Y, Gong Z, Ge B and Zang YQ: Berberine differentially modulates the activities of ERK, p38 MAPK and JNK to suppress Th17 and Th1 T cell differentiation in type 1 diabetic mice. J Biol Chem 284: 28420-28429, 2009.

14. Gu L, Li N, Yu W, Gong J, Li Q, Zhu W and Li J: Berberine reduces rat intestinal tight junction injury induced by ischemia-reperfusion associated with the suppression of inducible nitric oxide synthesis. Am J Chin Med 41: 1297-1312, 2013.

15. Chang W, Zhang M, Li J, Meng Z, Xiao D, Wei S, Chen L, Wang $C$ and Hatch GM: Berberine attenuates ischemia-reperfusion injury via regulation of adenosine-5'-monophosphate kinase activity in both non-ischemic and ischemic areas of the rat heart. Cardiovasc Drugs Ther 26: 467-478, 2012.

16. Curtis MJ and Walker MJ: Quantification of arrhythmias using scoring systems: An examination of seven scores in an in vivo model of regional myocardial ischaemia. Cardiovasc Res 22: 656-665, 1988.

17. Ravingerova T, Tribulova N, Slezak J and Curtis MJ: Brief, intermediate and prolonged ischemia in the isolated crystalloid perfused rat heart: Relationship between susceptibility to arrhythmias and degree of ultrastructural injury. J Mol Cell Cardio 27: 1937-1951, 1995.

18. Jia Y, Mo SJ, Feng QQ, Zhan ML, OuYang LS, Chen JC, Ma YX, Wu JJ and Lei WL: EPO-dependent activation of $\mathrm{PI} 3 \mathrm{~K} / \mathrm{Akt} / \mathrm{FoxO}$ a signalling mediates neuroprotection in in vitro and in vivo models of Parkinson's disease. J Mol Neurosci 53: 117-124, 2014. 
19. Zhang S, Lv JW and Yang P: Loss of dicer exacerbates cyclophosphamide-induced bladder overactivity by enhancing purinergic signaling. Am J Pathol 181: 937-946, 2012.

20. Sun X, Zhong J, Wang D, Xu J, Su H, An C, Zhu H and Yan J: Increasing glutamate promotes ischemia-reperfusion-induced ventricular arrhythmias in rats in vivo. Pharmacology 93: 4-9, 2014

21. Briest $F$ and Grabowski P: PI3K-AKT-mTOR-signaling and beyond: The complex network in gastroenteropancreatic neuroendocrine neoplasms. Theranostics 4: 336-365, 2014.

22. Shrimali D, Shanmugam MK, Kumar AP, Zhang J, Tan BK, Ahn KS and Sethi G: Targeted abrogation of diverse signal transduction cascades by emodin for the treatment of inflammatory disorders and cancer. Cancer Lett 341: 139-149, 2013.
23. Yin X, Zheng Y, Zhai X, Zhao X and Cai L: Diabetic inhibition of preconditioning- and postconditioning-mediated myocardial protection against ischemia/reperfusion injury. Exp Diabetes Res 2012: 198048, 2012.

24. Dong LY, Li S, Zhen YL, Wang YN, Shao X and Luo ZG: Cardioprotection of vitexin on myocardial ischemia/reperfusion injury in rat via regulating inflammatory cytokines and MAPK pathway. Am J Chin Med 41: 1251-1266, 2013. 